\title{
Identification of the Novel Pyroptosis-Related Gene Signature in Patients with Esophageal Adenocarcinoma
}

Ruijie Zeng ${ }^{1,2 \dagger}$, Shujie Huang ${ }^{2,3^{*} \dagger}$, Zewei Zhuo ${ }^{1}$, Huihuan $\mathrm{Wu}^{1}$, Weihong Sha ${ }^{1^{*}}$, Hao Chen ${ }^{1^{*}}$

1 Department of Gastroenterology, Guangdong Provincial People's Hospital, Guangdong Academy of Medical Sciences, Guangzhou 510080, China

${ }^{2}$ Shantou University Medical College, Shantou 515041, China

${ }^{3}$ Department of Thoracic Surgery, Guangdong Provincial People's Hospital, Guangdong Academy of Medical Sciences, Guangzhou 510080, China

*Correspondence: Prof. Hao Chen, Department of Gastroenterology, Guangdong Provincial People's Hospital, Guangdong Academy of Medical Sciences, Guangzhou 510080, China;

E-mail: chenhao@gdph.org.cn

Prof. Weihong Sha, Department of Gastroenterology, Guangdong Provincial People's Hospital, Guangdong Academy of Medical Sciences, Guangzhou 510080, China;

E-mail: shaweihong@gdph.org.cn

t: These authors contributed equally to this work 


\section{Abstract}

Esophageal adenocarcinoma (EAC) is a highly malignant type of digestive tract cancers with a poor prognosis despite therapeutic advances. Pyroptosis is an inflammatory form of programmed cell death, whereas the role of pyroptosis in EAC remains largely unknown. Herein, we identified a pyroptosis-related five-gene signature that was significantly correlated with the survival of EAC patients in The Cancer Genome Atlas (TCGA) cohort and an independent validation dataset. In addition, a nomogram based on the five-gene signature was constructed with novel prognostic values. Moreover, the genes in the pyroptosis-related signature, CASP1, GSDMB, IL1B, PYCARD, and ZBP1, might be involved in immune response and regulation of the tumor microenvironment. Our findings indicate that the fivegene signature provides insights into the involvement of pyroptosis in EAC progression, and is promising in the risk assessment as well as prognosis for EAC patients in clinical practice.

\section{Conflict of interest}

The authors declared no conflict of interest.

\section{Author Approvals}

The authors have seen and approved the manuscript, and that it hasn't been accepted or published elsewhere.

Keywords Pyroptosis, Esophageal adenocarcinoma, Prognosis, Tumor microenvironment 


\section{Introduction}

Esophageal cancer is one of the most common malignancies worldwide, accounting for approximately 604,100 new cases and 544,076 deaths per year over the world ${ }^{1}$. Esophageal adenocarcinoma (EAC) and esophageal squamous cell carcinoma (ESCC) composite the principle histologic subtypes of esophageal cancer, in which the incidence of EAC in western countries has increased dramatically in the last decades ${ }^{2}$. Despite therapeutic advances in surgery, radiotherapy, chemotherapy, and targeted drugs, the 5-year survival of esophageal cancer remains less than $20 \%{ }^{3}$. In consequence, biomarkers and effective models are urgently needed to predict the prognosis of EAC and provide insights into targeted therapy.

Pyroptosis is a pro-inflammatory form of regulated cell death, relying on the enzymatic activity of inflammatory proteases that belong to the caspase family ${ }^{4}$. Pyroptosis is featured with swift plasma-membrane rupture and subsequent release of pro-inflammatory intracellular contents, which is distinct from apoptosis ${ }^{5}$. Studies evaluating the role of pyroptosis in neurological, infectious, autoimmune, cardiovascular and oncologic disorders have been emerging in recent years ${ }^{6}$. Activation of the canonical inflammasome pathway is the basis of pyroptosis, in which pattern-recognition receptors (PRRs), for example, Toll-like receptors (TLRs), nucleotide-binding oligomerization domain-like receptor (NLRs) and absent in melanoma 2 like-receptor (ALRs) recognize pathogen-associated molecular patterns (PAMPs) or nonpathogen-related damage-associated molecular patterns (DAMPs) to activate inflammasomes and facilitate caspase- 1 activation ${ }^{7}$. Direct activation of caspase-4/5/11 under lipopolysaccharide (LPS) is involved in the noncanonical pyroptosis pathway, which is independent of the inflammasome complex ${ }^{8}$. The gasdermin (GSDM) family proteins serve 
as the main mediators of pyroptosis, which are proteolytically activated by proteases and induce the formation of plasma membrane pores, leading to cell swelling and lysis ${ }^{9,10}$. Due to the pivotal role of GSDM family proteins, pyroptosis is defined by some researchers as gasdermin-mediated programmed cell death ${ }^{11}$.

The role of pyroptosis has been explored in various types of cancer. GSDME is silenced in the majority of cancer cells, whereas expressed in various normal tissues ${ }^{12}$. The knockdown of GSDMD inhibits the proliferation of non-small cell lung cancer (NSCLC) cells by intrinsic mitochondrial apoptotic pathways ${ }^{13}$. The knock-out of inflammatory vesicle-related genes in vivo demonstrates the tendency to develop colon cancers ${ }^{14}$. Metformin induces GSDMD-mediated pyroptosis and serves as an alternative treatment for therapy-refractory ESCC. Moreover, the PLK1 kinase inhibitor BI2536 sensitizes ESCC cells to cisplatin by inducing pyroptosis.

However, despite research is emerging in ESCC, the role of pyroptosis in esophageal cancer remains largely unknown, and none of the previous publications have comprehensively evaluated the pyroptosis-related genes in EAC. Therefore, we performed a comprehensive evaluation of pyroptosis-related genes in EAD, in order to develop a pyroptosis-gene-based modality to predict the prognosis of the patients, and provide insights into the correlations between pyroptosis and tumor immune microenvironment.

\section{Methods}

\section{Datasets}

The RNA-sequencing (RNA-seq) data of 87 patients (78 with EAD; 9 normal samples) and the 
corresponding clinical information from The Cancer Genome Atlas (TCGA) database were retrieved on May $20^{\text {th }}, 2021$ (https://portal.gdc.cancer.gov/repository). The RNA-seq data and clinical features of the validation cohort were downloaded from the Gene Expression Omnibus (GEO) database (https://www.ncbi.nlm.nih.gov/geo/, ID: GSE13898). Compared to the followup time of the TCGA cohort, that of the GSE13898 cohort was shorter (up to 4.9 years).

\section{Identification of differentially expressed genes (DEGs) in pyroptosis-related gene set}

The 58 pyroptosis-related genes were derived from prior literature and the Gene Ontology (GO) term pyroptosis (ID: GO0070269; Supplementary Table S1) ${ }^{7,15,16,17}$. Normalization of expression data to fragment per kilobase million (FPKM) values was performed in both datasets. The package "limma" was used to explore DEGs with the threshold of $P$ value $<0.05$ 18. Protein-protein interaction (PPI) networks were created by Search Tool for the Retrieval of Interacting Genes (STRING) and the "igraph" package ${ }^{19,20 .}$

Development and validation of the pyroptosis-correlated gene prediction model for prognosis

Cox regression analysis was employed to evaluate the value of pyroptosis-related genes for prognosis. The DEGs were identified for further analysis. The LASSO Cox regression analysis was employed to construct a refined model for prognosis using the R package "glmnet" ${ }^{21}$. The calculation of the risk score was performed using the following formula: risk score = $\sum_{(n=1)}^{i}$ Coef $i * X i$ (Coef $\mathrm{i}$ indicates the coefficient, and $\mathrm{Xi}$ indicates the gene expression levels after standardization). The EAC patients were classified into low- and high-risk groups based 
on the median risk score, and Kaplan-Meier analysis was used to compare the overall survival (OS) between the two groups. Principal component analysis (PCA) was used to assess the separability of the two groups by the "prcomp" function. The R packages "survival", "survminer", "timeROC" and "riskRegression" were utilized for receiver operating characteristic (ROC) curve graphing and area under curve (AUC) calculation for 1,2 and 5 years ${ }^{22,23,24,25}$. A nomogram model with clinical features including stage and risk score was constructed by the R packages "rms", "foreign" and "survival" 22, 26, 27. The calibration curve and detrended correspondence analysis (DCA) were performed using the "rms" package ${ }^{26}$. An EAC cohort (GSE13898) from the GEO database was used for validation, and the risk score was calculated by the same methods described above to divide the cohort into two subgroups (low risk and high risk).

\section{Prognostic analysis of the variables}

Clinical data (gender and stage) was extracted of patients in the TCGA cohort and the GSE13898 cohorts. Variables including gender, stage and risk score were analyzed in the regression model by univariate and multivariate Cox regression analysis.

\section{Enrichment analysis}

Patients with EAC in the TCGA cohort were divided into two groups based on the median risk score. The DEGs between the low- and high-risk groups were extracted by $|\log 2 \mathrm{FC}| \geq 1$ and $P$ value $<0.05$. GO and Kyoto Encyclopedia of Genes and Genomes (KEGG) pathway enrichment was performed by the R package "clusterProfiler", and the results were visualized 
using the "GOplot" package ${ }^{28,29}$.

\section{Tumor microenvironment analysis}

The Tumor Immune Estimation Resource (TIMER) database (https://cistrome.shinyapps.io/timer/) was utilized to assess the correlation between tumorinfiltrating immune cells and expressions of selected genes ${ }^{30}$. Estimation Resource (TIMER) was used to compare the immune scores of the four subtypes. The CIBERSORT algorithm was used to further explore the composition and differences in the fraction of 22 immune cell types between two subgroups classified by risk scores ${ }^{31}$.

\section{Statistical analysis}

Statistical analyses were performed by $R$ (version 4.1 .0 ). Student's t-test was applied to compare the differences in gene expression between tumor and normal tissues, while categorical variables were compared using Pearson chi-square test. The OS of patients between low- and high-risk groups were compared by the Kaplan-Meier method with log-rank test. The Cox regression analysis was performed to evaluate the independent prognostic factors for survival. The Wilcoxon test was used to compare the immune cell infiltration between groups.

\section{Results}

Identification of DEGs between EAC and normal tissues

The expression levels of 58 pyroptosis-correlated genes were examined in the TCGA data of 
$78 \mathrm{EAC}$ and 9 normal tissues. Ten DEGs were identified $(|\log 2 \mathrm{FC}| \geq 1$ and $P$ value $<0.05)$, and all of them (CASP1, CASP5, GSDMB, GZMB, IL1B, NLRP6, PYCARD, TNF, TREM2, ZBP1) were upregulated in the tumor group. The expression profiles of DEGs were demonstrated in Fig. 1A (red color represents higher expression level; blue color represents lower expression level). Fig. 1B showed the correlation network of DEGs in the TCGA data. The PPIs of DEGs were presented in Fig. 1C, in which the interaction score was set as 0.4.

\section{Construction of prognostic model based on DEGs}

A total of 65 EAC patients with available survival data were included in our study. Univariate Cox regression analysis was initially performed to assess the prognostic value of DEGs (Fig. 2A). Among them, 6 genes (CASP1, CASP5, GSDMB, IL1B, PYCARD and ZBP1) were with the $P$ value $<0.2$, and higher expressions of CASP1, CASP5, IL1B were associated with increased risk (HR > 1), while upregulated expressions of GSDMB, PYCARD, ZBP1 were correlated with lower risk $(H R<1)$. Subsequently, LASSO Cox regression analysis retrieved 5 genes for prognostic model construction based on the optimum $\lambda$ value (Fig. 2B, 2C). The calculation of risk score was as follows: Risk score $=\left(0.042{ }^{*} \operatorname{expCASP} 1\right)+(-0.025$ * $\exp G S D M B)+(0.021 * \operatorname{explL1B})+(-0.037$ * $\exp P Y C A R D)+(-0.243+\exp Z B P 1)$ According to the calculated median risk score, 65 patients were divided into two groups (32 in the high-risk group and 33 in the low-risk group), and the clinical information was shown in

Fig. 3A. The PCA analysis illustrated that patients were well divided into two clusters (Fig. 3B). The distributions of risk score and survival time were shown in Fig. 3C, 3D. The OS of the high-risk group was significantly worse than that of the low-risk group $(P=0.0012$, Fig. 
3E). ROC analysis of the risk model indicated that the AUC for 1-year, 2-year and 5-year survival was $0.708,0.815$ and 0.952 , respectively (Fig. 3F, 3G, 3H). Both of the univariate and multivariate Cox regression analyses showed that the pyroptosis-related gene signature independently predicted the prognosis of EAC patients (Fig. 3I, 3J).

\section{Verification of the gene signature by the external dataset}

Information of 60 EAC patients from the GSE13898 dataset of GEO with available survival data was used for validation of the 5-gene signature. The patients were subdivided into the low- and high-risk group respectively as described above. PCA illustrated well separation of patients between the two groups (Fig. 4A). The distribution of risk score and survival time was demonstrated in Fig. 4B, 4C. Patients in the low-risk group were with significantly higher survival rates than those in the high-risk group $(P=0.003$; Fig. 4D). According to the ROC curve, the 1-year and 2-year survival prediction models were with the AUC of 0.678 and 0.663 (Fig. 4E, 4F), respectively, while the 5-year survival prediction model could not be generated due to insufficient data. The risk score in our model could also serve as an independent prognostic factor in the validation cohort (Fig. 4G, 4H).

\section{Construction of nomogram based on the gene signature and clinical data}

In order to more precisely predict the prognosis of EAC patients, TNM stage was used to construct a nomogram model as shown in Fig. 5A. The AUCs of the nomogram for predicting 1-year, 2-year and 5-year survival were 0.722, 0.884, 1.00, respectively (Fig. 5B, 5C, 5D). The calibration curve indicated an ideal prediction of the nomogram (Fig. 5E). Fig. 5F showed 
that when the nomogram-predicted probability was ranged from $15 \%$ to $80 \%$, the nomogram provided extra value relative to the treat-all-patients scheme or the treat-none scheme.

\section{Differential expression analysis}

A total of 527 DEGs between the low- and high-risk groups were extracted according to the threshold described above. A total of 310 genes were downregulated in the high-risk group, while 217 genes were upregulated in the low-risk group (Table S2). On the basis of the DEGs, GO enrichment and KEGG pathway analyses were performed. The results from GO enrichment analysis demonstrated that the DEGs were mainly associated with the regulation of cytokine production, cytokine activity and humoral immune response pathways (Fig. 6A, B). KEGG pathway analysis showed that the DEGs were principally associated with the cytokinecytokine receptor interaction and IL-17 signaling pathways (Fig. 6C, 6D).

\section{Exploration of association with immune status of EAC}

To explore the correlation between the selected pyroptosis-related genes and gene-based signature with the immune microenvironment of EAC, analysis by TIMER database for each gene was initially performed. The results indicated that ZBP1 expression was most significantly correlated with the infiltration signature of esophageal cancer, in which infiltrations of $\mathrm{B}$ cells (correlation coefficient $=0.366, P=4.72 \mathrm{e}-07$ ) and CD4 $+\mathrm{T}$ cells (correlation coefficient $=0.381, P=1.41 \mathrm{e}-07$ ) were with the most remarkable correlations (Fig. 7A, Supplementary Fig. S1). In addition, somatic copy number alterations of ZBP1 were correlated with the infiltration levels of B cells, CD8+ T cells, macrophages and dendritic cells. 
The variations in the abundance of immune cell infiltration between low- and high-risk groups were further explored. The immune cells were analyzed in the TCGA(Supplementary Table S3) and GSE13898 cohorts (Supplementary Table S4). The overview of immune cell compositions was illustrated in Fig. 8A for the TCGA cohort, and Fig. 8B for the GSE13898 cohort. The high-risk group in the TCGA cohort possessed significantly higher infiltration levels of M2 macrophages, activated mast cells and eosinophils, whereas the infiltration levels of plasma cells were significantly lower (Fig. 8C, 8D). By contrast, the infiltration levels of memory B cells and M1 macrophages were upregulated in the high-risk group of the GSE13898 cohort, while those of naïve B cells were significantly downregulated (Fig. 8E, 8F).

\section{Discussion}

Cell death serves as an essential barrier against the development of cancer, and pyroptosis is one of the major forms of programmed cell death ${ }^{32}$. Mediated by the gasdermin family, pyroptosis is accompanied by immune and inflammatory responses ${ }^{7}$. Emerging evidence has indicated the involvement of pyroptosis in cancer, while the dual role of pyroptosis has attracted the researchers' attention ${ }^{7}$. However, the role of pyroptosis in EAC remains largely unclear. In the present study, we comprehensively evaluated the pyroptosis-related gene profiles in EAC, and constructed a novel 5-gene risk signature (CASP1, GSDMB, IL1B, PYCARD, ZBP1) by LASSO Cox regression analysis. The 5-gene signature showed well performance for predicting EAC prognosis in both the internal and external validation cohorts. Further enrichment analyses revealed that the DEGs between the low- and high-risk groups were correlated with immune-related pathways. Tumor immune microenvironment analyses 
indicated that high-risk patients had decreased levels of infiltrating active immune cells and higher proportions of quiescent immune-cell infiltration.

Caspase 1 that encoded by CASP1 is a member of the caspase family, which is activated by inflammasomes and induces pyroptosis ${ }^{33}$. As tumor suppressor, the expressions of Caspase 1 are lower in various cancer tissues ${ }^{34}$. Caspase 1 activation-mediated pyroptosis suppresses glioma cell growth and metastasis ${ }^{35}$. Gasdermin B (GSDMB) belongs to the GSDM family and is more broadly expressed compared to other GSDM family members ${ }^{36}$. The cleavage of GSDMB induced by lymphocyte-derived granzyme A triggers pyroptosis ${ }^{37}$. By contrast, GSDMB is found highly expressed in several human cancer tissues, whereas the specific functions and mechanisms remain unclear ${ }^{38}$. Interleukin 1 Beta (IL-1 $\beta$ ) is a proinflammatory cytokine involved in pyroptosis. CASP-1 directly cleaves GSMD and precursor cytokines into pro-IL-1 $\beta$, which initiates pyroptosis and maturation of IL-1 $\beta$, respectively ${ }^{39}$. IL-1 $\beta$ has pro-tumorigenic effects by promoting proliferation, migration and metastasis ${ }^{40}$. Apoptosis-associated speck-like protein containing a CARD (ASC) is encoded by PYCARD gene and contains a caspase activation and recruitment domain (CARD) for binding and facilitating the activation of caspase $1^{5}$. The dual role of the inflammasome adaptor ASC is identified in cancer cells, as ASC has both pro-apoptotic and pro-inflammatory functions ${ }^{41}$. Z-DNA-binding protein 1 (ZBP1)-NLR Family Pyrin Domain Containing 3 (NLRP3) is critical in inducing pyroptosis by leading to cytokine maturation and GSDMD cleavage ${ }^{42}$. Deletion of ZBP1 blocks tumor necroptosis and inhibits metastasis in breast cancer cells ${ }^{43}$. However, the effects and mechanisms of ZBP1 in tumors are poorly understood. In the current study, all of the 5 genes were upregulated in tumor samples compared to normal tissues, 
which is consistent with previous publications. Intriguingly, GSDMB, PYCARD and ZBP1 were identified as down-regulated in the high-risk populations. It could be due to the pro-pyroptotic effect of GSDMB and the dual role of ASC in cancer cells. Therefore, pyroptotic-related genes might exert different effects in tumor development and progression depending on the biological context. Since the research of ZBP1 in cancer is in its infancy, it is of importance for further exploration for its role and downstream-regulated pathways, especially for the involvement in the tumor microenvironment.

Crosstalks between the critical modes of programmed cell death exist, including apoptosis, pyroptosis and necroptosis, and part of their pathways are overlapping. For example, CASP1, IL1B and PYCARD are well-known regulators in the apoptotic pathways. It is inevitable that these pathways might have interactions as the tumor develops and progresses. Based on our enrichment analysis, immune response-correlated pathways are dysregulated in different risk groups, which indicates that inflammatory responses can be induced by pyroptotic cell death.

Nomograms are promising for use in clinical practice for evaluating the prognosis of EAC patients, in which the survival can be predicted using specific parameters. As indicated by the ROC curves, the nomogram demonstrates high predictive accuracy and sensitivity, especially for the prediction of 5-year survival. Compared to the conventional TNM staging and previously developed ferroptosis-related gene-signature in EAC ${ }^{44}$, the pyroptosis-related gene signature-based nomogram, which integrates gene expression profiles and clinical parameters, more effectively predicts the prognosis of EAC patients. The use of nomogram based on integrated information can facilitate the prediction of prognosis, clinical decision- 
making and patient counseling ${ }^{45}$.

Current studies on lymphocytes in tumor immunity predominantly focus on T cells, while the protective effect of $B$ cells has also been revealed ${ }^{46}$. By contrast, Mast cells have been reported to induce cancer growth ${ }^{47}$. Activated T cells, natural killer cells and macrophages are

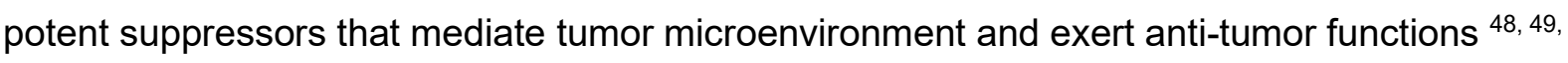
${ }^{50}$. Although some of the comparisons were not statistically different and might be contributed by the limited number of samples in both cohorts, accumulation of immune cells that promote cancer in the tumor microenvironment was generally observed in the high-risk group in both the TCGA and GEO cohort, while the compositions of tumor-protective immune cells were reduced compared to the low-risk group. In conclusion, the poor survival in the high-risk patients with EAC might be contributed by suppressed levels of anti-tumor immunity and alterations in the tumor microenvironment.

The strength of our study is that a systemic analysis was performed based on the TCGA and GEO cohorts, and the pyroptosis-related genes were assessed for the first time. Limitations also exist in our study. Due to the nature of publicly available dataset, the cases of EAC patients were limited, and the data of therapeutic responses were not available (e.g., chemotherapy, radiotherapy, immunotherapy). Specific mechanisms that pyroptosis-related genes regulate EAC development and progression remain to be explored. Large-scale and well-designed clinical studies for validation of our prediction model need to be performed. Despite the limitations, our study has provided a comprehensive overview of pyroptosisrelated gene profiles in EAC.

In summary, we identified differentially expressed pyroptosis-related genes and 
developed a novel five-gene pyroptosis signature that significantly correlates with the survival of EAC patients. The pyroptosis-based signature is an independent prognostic factor and performs better than the TNM stage, which is promising for clinical application. Moreover, the genes in the pyroptosis-based signature might regulate anti-tumor immunity in tumor microenvironment, and further studies are warranted.

\section{Acknowledgement}

The authors thank Prof. Ju-Seog Lee for kindly providing the data of the GSE13898 cohort.

\section{Funding}

This work is supported by the National Natural Science Foundation of China (81300279, 81741067), the Natural Science Foundation for Distinguished Young Scholars of Guangdong Province (2021B1515020003), the Climbing Program of Introduced Talents and High-level Hospital Construction Project of Guangdong Provincial People's Hospital (DFJH201923, DFJH201803, KJ012019099).

\section{Conflict of interest}

The authors declared no conflict of interest.

\section{Data availability}

The datasets are available in TCGA database (https://portal.gdc.cancer.gov) and GEO database (https://www.ncbi.nlm.nih.gov/gds/, accession number: GSE13898). R code used in 
bioRxiv preprint doi: https://doi.org/10.1101/2021.07.05.451093; this version posted July 5, 2021. The copyright holder for this preprint (which was not certified by peer review) is the author/funder. All rights reserved. No reuse allowed without permission.

this study are available from the corresponding author upon reasonable request. 


\section{Reference}

1. Sung H, et al. Global Cancer Statistics 2020: GLOBOCAN Estimates of Incidence and Mortality Worldwide for 36 Cancers in 185 Countries. CA Cancer J Clin 71, 209-249 (2021).

2. Klingelhöfer D, Zhu Y, Braun M, Brüggmann D, Schöffel N, Groneberg DA. A world map of esophagus cancer research: a critical accounting. J Transl Med 17, 150 (2019).

3. Alsop BR, Sharma P. Esophageal Cancer. Gastroenterol Clin North Am 45, 399-412 (2016).

4. Vande Walle L, Lamkanfi M. Pyroptosis. Curr Biol 26, R568-r572 (2016).

5. Bergsbaken T, Fink SL, Cookson BT. Pyroptosis: host cell death and inflammation. Nat Rev Microbiol 7, 99-109 (2009).

6. Yu P, Zhang X, Liu N, Tang L, Peng C, Chen X. Pyroptosis: mechanisms and diseases. Signal Transduction and Targeted Therapy 6, 128 (2021).

7. Xia X, et al. The role of pyroptosis in cancer: pro-cancer or pro-"host"? Cell Death Dis 10, 650 (2019).

8. Shi J, et al. Inflammatory caspases are innate immune receptors for intracellular LPS. Nature 514, 187-192 (2014).

9. Van Opdenbosch N, Lamkanfi M. Caspases in Cell Death, Inflammation, and Disease. Immunity 50, 1352-1364 (2019).

10. Tsuchiya K. Inflammasome-associated cell death: Pyroptosis, apoptosis, and physiological implications. Microbiol Immunol 64, 252-269 (2020).

11. Shi J, Gao W, Shao F. Pyroptosis: Gasdermin-Mediated Programmed Necrotic Cell Death. Trends Biochem Sci 42, 245-254 (2017).

12. Wang $\mathrm{Y}$, et al. Chemotherapy drugs induce pyroptosis through caspase-3 cleavage of a gasdermin. Nature 547, 99-103 (2017).

13. Gao J, et al. Downregulation of GSDMD attenuates tumor proliferation via the intrinsic mitochondrial apoptotic pathway and inhibition of EGFR/Akt signaling and predicts a good prognosis in non-small cell lung cancer. Oncol Rep 40, 1971-1984 (2018).

14. Dupaul-Chicoine J, et al. Control of intestinal homeostasis, colitis, and colitis-associated colorectal cancer by the inflammatory caspases. Immunity 32, 367-378 (2010).

15. Karki R, Kanneganti TD. Diverging inflammasome signals in tumorigenesis and potential 
targeting. Nat Rev Cancer 19, 197-214 (2019).

16. Wang B, Yin Q. AIM2 inflammasome activation and regulation: A structural perspective. $J$ Struct Biol 200, 279-282 (2017).

17. Man SM, Kanneganti TD. Regulation of inflammasome activation. Immunol Rev 265, 6-21 (2015).

18. Ritchie ME, et al. limma powers differential expression analyses for RNA-sequencing and microarray studies. Nucleic Acids Res 43, e47 (2015).

19. Szklarczyk D, et al. STRING v11: protein-protein association networks with increased coverage, supporting functional discovery in genome-wide experimental datasets. Nucleic Acids Res 47, D607-d613 (2019).

20. Csardi G, Nepusz T. The igraph software package for complex network research. InterJournal, complex systems 1695, 1-9 (2006).

21. Friedman J, Hastie T, Tibshirani R. Regularization paths for generalized linear models via coordinate descent. Journal of statistical software 33, 1 (2010).

22. Therneau TM, Lumley T. Package 'survival'. R Top Doc 128, 28-33 (2015).

23. Kassambara A, Kosinski M, Biecek P, Fabian S. survminer: Drawing Survival Curves using'ggplot2'. R package version 03 1, (2017).

24. Blanche P, Dartigues JF, Jacqmin - Gadda H. Estimating and comparing time - dependent areas under receiver operating characteristic curves for censored event times with competing risks. Statistics in medicine 32, 5381-5397 (2013).

25. Ozenne B, Sørensen AL, Scheike T, Torp-Pedersen C, Gerds TA. riskRegression: predicting the risk of an event using Cox regression models. The $R$ Journal 9, 440-460 (2017).

26. Harrell Jr FE, Harrell Jr MFE, Hmisc D. Package 'rms'. Vanderbilt University 229, (2017).

27. Team RC, et al. Package 'foreign'. (2020).

28. Yu G, Wang L-G, Han Y, He Q-Y. clusterProfiler: an R package for comparing biological themes among gene clusters. Omics: a journal of integrative biology 16, 284-287 (2012).

29. Walter W, Sánchez-Cabo F, Ricote M. GOplot: an R package for visually combining expression data with functional analysis. Bioinformatics 31, 2912-2914 (2015).

30. Li T, et al. TIMER: A Web Server for Comprehensive Analysis of Tumor-Infiltrating Immune 
Cells. Cancer Res 77, e108-e110 (2017).

31. Chen B, Khodadoust MS, Liu CL, Newman AM, Alizadeh AA. Profiling tumor infiltrating immune cells with CIBERSORT. In: Cancer systems biology). Springer (2018).

32. Bertheloot D, Latz E, Franklin BS. Necroptosis, pyroptosis and apoptosis: an intricate game of cell death. Cellular \& Molecular Immunology 18, 1106-1121 (2021).

33. Miao EA, Rajan JV, Aderem A. Caspase-1-induced pyroptotic cell death. Immunol Rev 243, 206-214 (2011).

34. Shalini S, Dorstyn L, Dawar S, Kumar S. Old, new and emerging functions of caspases. Cell Death \& Differentiation 22, 526-539 (2015).

35. Jiang Z, et al. miRNA-214 inhibits cellular proliferation and migration in glioma cells targeting caspase 1 involved in pyroptosis. Oncology research 25, 1009 (2017).

36. Saeki N, et al. Distinctive expression and function of four GSDM family genes (GSDMA-D) in normal and malignant upper gastrointestinal epithelium. Genes Chromosomes Cancer $\mathbf{4 8}$, 261-271 (2009).

37. Zhou Z, et al. Granzyme A from cytotoxic lymphocytes cleaves GSDMB to trigger pyroptosis in target cells. Science 368, (2020).

38. Li L, Li Y, Bai Y. Role of GSDMB in Pyroptosis and Cancer. Cancer Manag Res 12, 3033-3043 (2020).

39. Man SM, Karki R, Kanneganti TD. Molecular mechanisms and functions of pyroptosis, inflammatory caspases and inflammasomes in infectious diseases. Immunol Rev 277, 61-75 (2017).

40. Rébé C, Ghiringhelli F. Interleukin-1 $\beta$ and Cancer. Cancers (Basel) 12, (2020).

41. Protti MP, De Monte L. Dual Role of Inflammasome Adaptor ASC in Cancer. Front Cell Dev Biol 8, 40 (2020).

42. Zheng M, Kanneganti TD. The regulation of the ZBP1-NLRP3 inflammasome and its implications in pyroptosis, apoptosis, and necroptosis (PANoptosis). Immunol Rev 297, 26-38 (2020).

43. Baik JY, et al. ZBP1 not RIPK1 mediates tumor necroptosis in breast cancer. Nat Commun 12, 2666 (2021).

44. Zhu L, et al. Identification the ferroptosis-related gene signature in patients with esophageal 
adenocarcinoma. Cancer Cell Int 21, 124 (2021).

45. Bobdey S, Mair M, Nair S, Nair D, Balasubramaniam G, Chaturvedi P. A Nomogram based prognostic score that is superior to conventional TNM staging in predicting outcome of surgically treated T4 buccal mucosa cancer: Time to think beyond TNM. Oral Oncol 81, 10-15 (2018).

46. Wang S-s, Liu W, Ly D, Xu H, Qu L, Zhang L. Tumor-infiltrating B cells: their role and application in anti-tumor immunity in lung cancer. Cellular \& molecular immunology 16, 6-18 (2019).

47. Maciel TT, Moura IC, Hermine O. The role of mast cells in cancers. F1000Prime Rep 7, 09 (2015).

48. Nurieva RI, et al. Function of $\mathrm{T}$ follicular helper cells in anti-tumor immunity.). Am Assoc Immnol (2019).

49. Lin YC, et al. Activated but not resting regulatory $T$ cells accumulated in tumor microenvironment and correlated with tumor progression in patients with colorectal cancer. Int J Cancer 132, 1341-1350 (2013).

50. Cózar B, Greppi M, Carpentier S, Narni-Mancinelli E, Chiossone L, Vivier E. Tumor-infiltrating natural killer cells. Cancer Discovery 11, 34-44 (2021). 


\section{Figure Legends}

Figure 1. Expressions of 10 differentially expressed pyroptosis-related genes and interaction. A. Heatmap of gene expression between the tumor ( $\mathrm{T}$, red color) and normal $(\mathrm{N}$, blue color) group. Higher expression: red color. Lower expression: blue color. B. Correlation network of 10 differentially expressed pyroptosis-related genes (red: positive correlation; blue: negative correlation; deeper color indicates higher strength of relevance). C. Protein-protein interaction (PPI) network of proteins encoded by selected genes.

Figure 2. Construction of pyroptosis-related gene risk signature in TCGA cohort. A. Univariate cox regression analysis of overall survival (OS) for the selected genes. B. LASSO regression of the 10 selected genes. C. Cross-validation for tuning the parameter selection in lasso regression.

Figure 3. Prediction of prognosis using the pyroptosis-related five-gene signature in TCGA cohort. A. Heatmap of 5 selected gene expressions with clinical features ordered by risk score (red: higher expression; blue: lower expression). B. Principal component analysis (PCA) of the risk groups. C. Distribution of patients according to risk scores. D. Survival time and status of patients. E. Kaplan-Meier curves for the survival of patients in the low- and highrisk groups. F, G, H. Receiver operating characteristic (ROC) curve for 1-, 2- and 5-year survival of patients. I. Univariate Cox analysis. J. Multivariate Cox analysis.

Figure 4. Validation of the pyroptosis-related five-gene signature in GSE13898 cohort.

A. Principal component analysis (PCA) of the risk groups. B. Distribution of patients according to risk scores. C. Survival time and status of patients. D. Kaplan-Meier curves for the survival of patients in the low- and high-risk groups. E, F. Receiver operating characteristic (ROC) 
curve for 1-, 2- and 5-year survival of patients. G. Univariate Cox analysis. H. Multivariate Cox analysis.

Figure 5. Construction of nomogram based on the pyroptosis-related five-gene signature. A. Nomogram for predicting 1, 2, 5-year survival of EAC patients. B, C, D. Receiver operating characteristic (ROC) curve evaluating the efficiency of nomogram for 1-, 2- and 5year survival of patients. E. Calibration curve of nomogram. F. Decision curve analysis (DCA) curve of the nomogram.

Figure 6. Functional enrichment analysis of differentially regulated genes (DEGs) between low- and high-risk groups. A, B. DEGs and pathways clustered by GO pathway enrichment analysis. C, D. DEGs and pathways clustered by KEGG pathway enrichment analysis.

Figure 7. Correlations between immune cells and selected genes. A. ZBP1 and immune cells in esophageal cancer. B. Mutants (Arm-level Deletion, Arm-level Gain and High Amplication) of ZBP1 compared with Diploid/Normal in esophageal cancer.

Figure 8. Tumor microenvironment immune cell composition in low- and high-risk groups. A. Overview of immune cell composition in TCGA cohort. B. Overview of immune cell composition in GSE13898 cohort. C, D. Differences in immune cell composition in TCGA cohort classified by risk groups by Wilcoxon test. E, F. Differences in immune cell composition in GSE13898 cohort classified by risk groups by Wilcoxon test. 


\section{Supplementary Information}

Supplementary Figure S1. Correlations between immune cells and other genes in the pyroptosis signature.

Supplementary Table S1. Pyroptosis-related genes included in analysis.

Supplementary Table S2. Differentially expressed genes between the risk groups.

Supplementary Table S3. Immune cell composition in the TCGA cohort divided by risk groups.

Supplementary Table S4. Immune cell composition in the GSE13898 cohort divided by risk groups. 
Figure 1

A

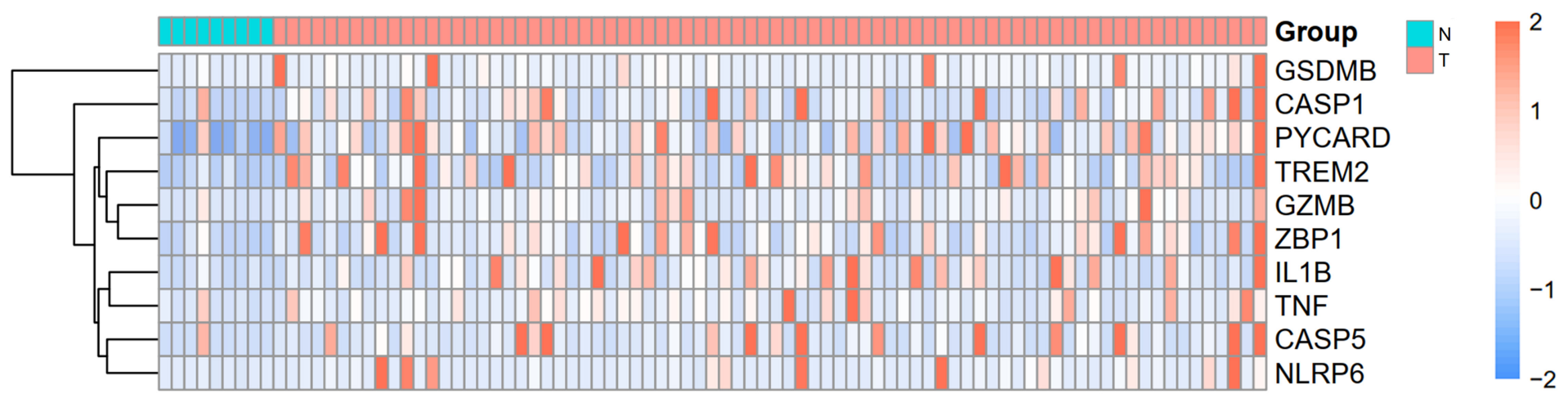

B

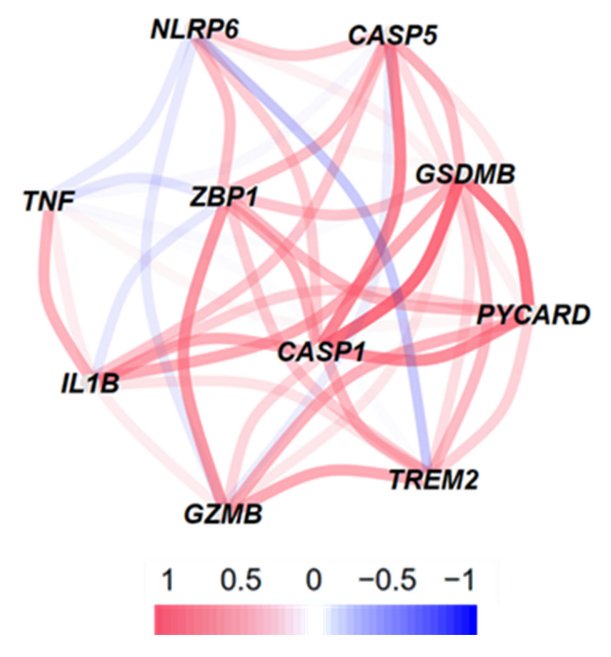

C

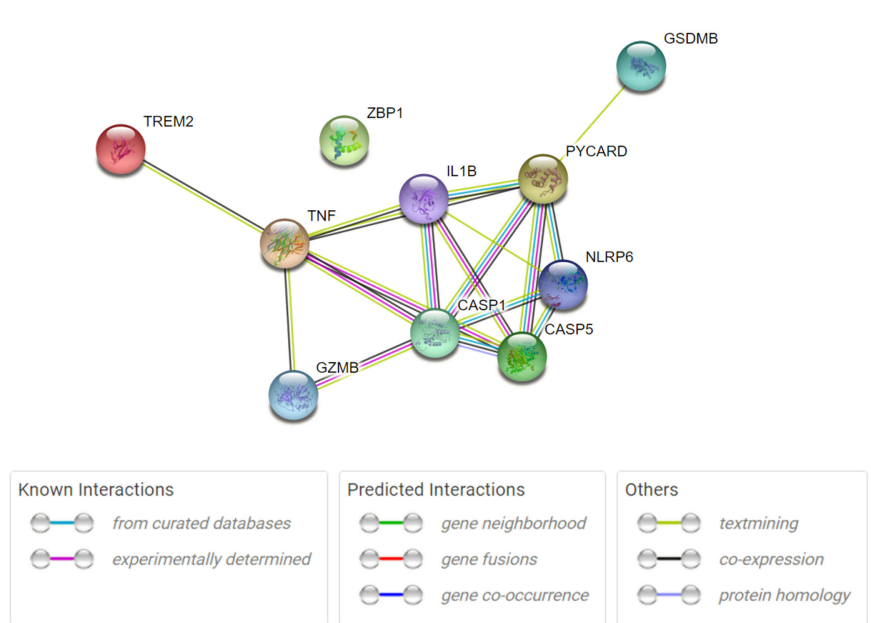


Figure 2

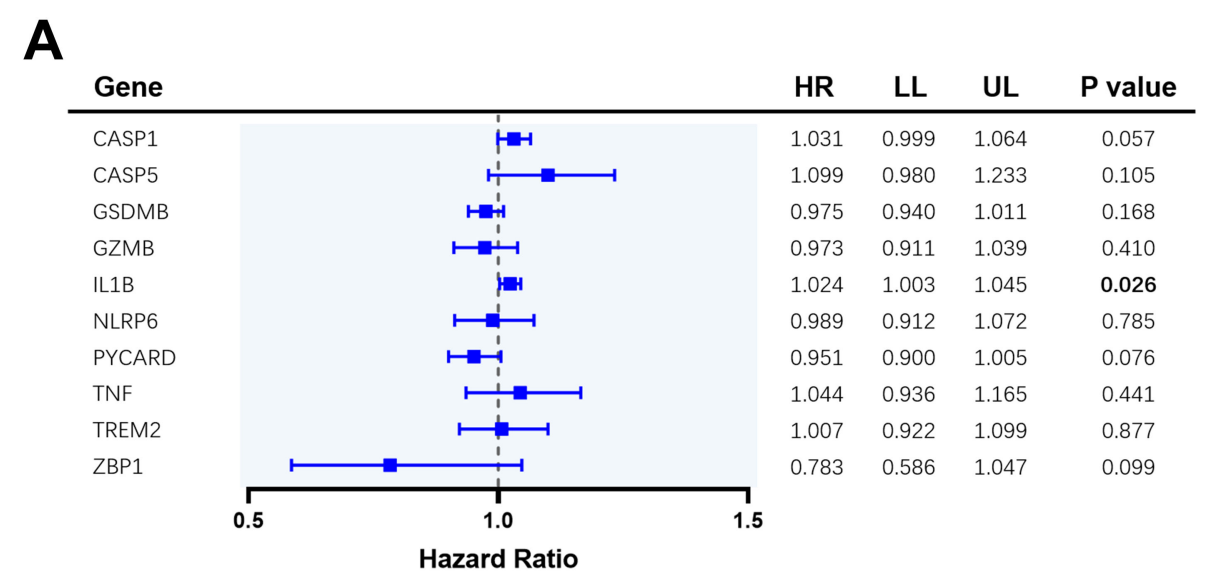

B

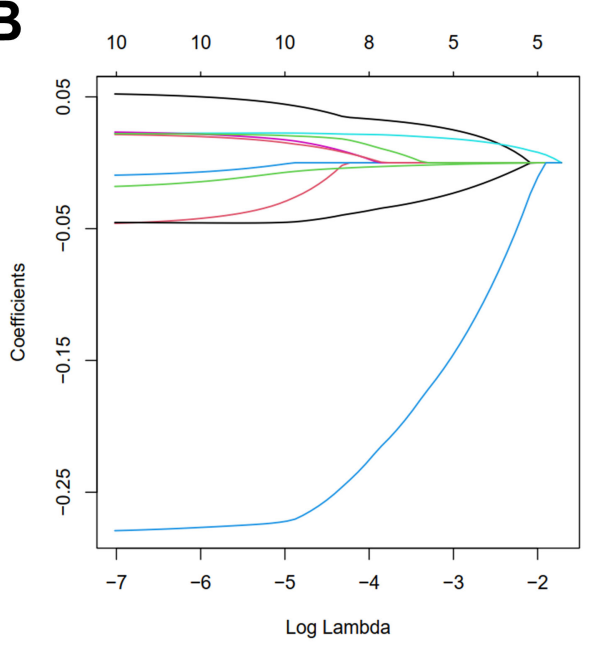

C

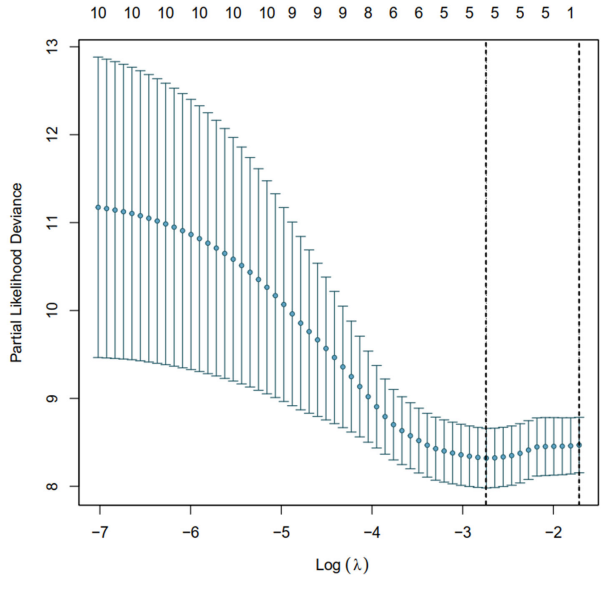


Figure 3

A

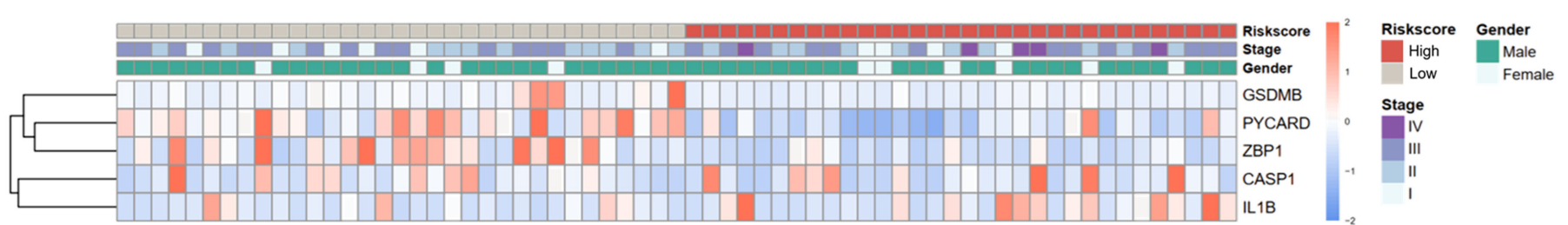

B

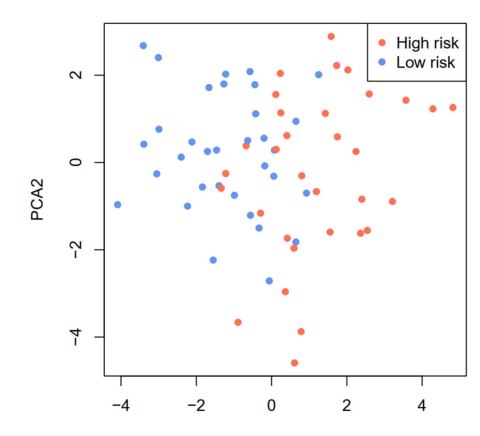

E
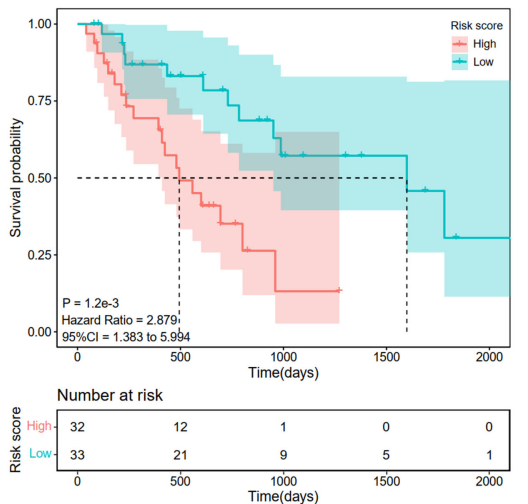

I
C

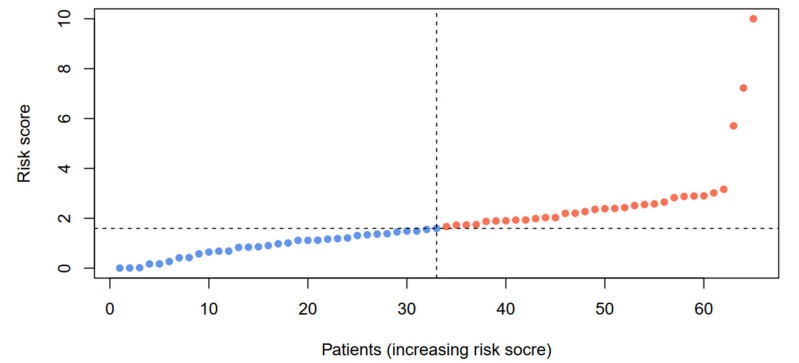

F

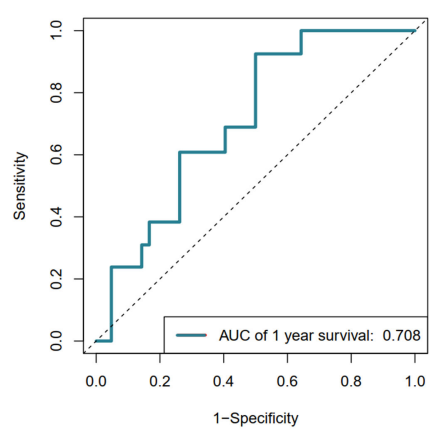

G
D
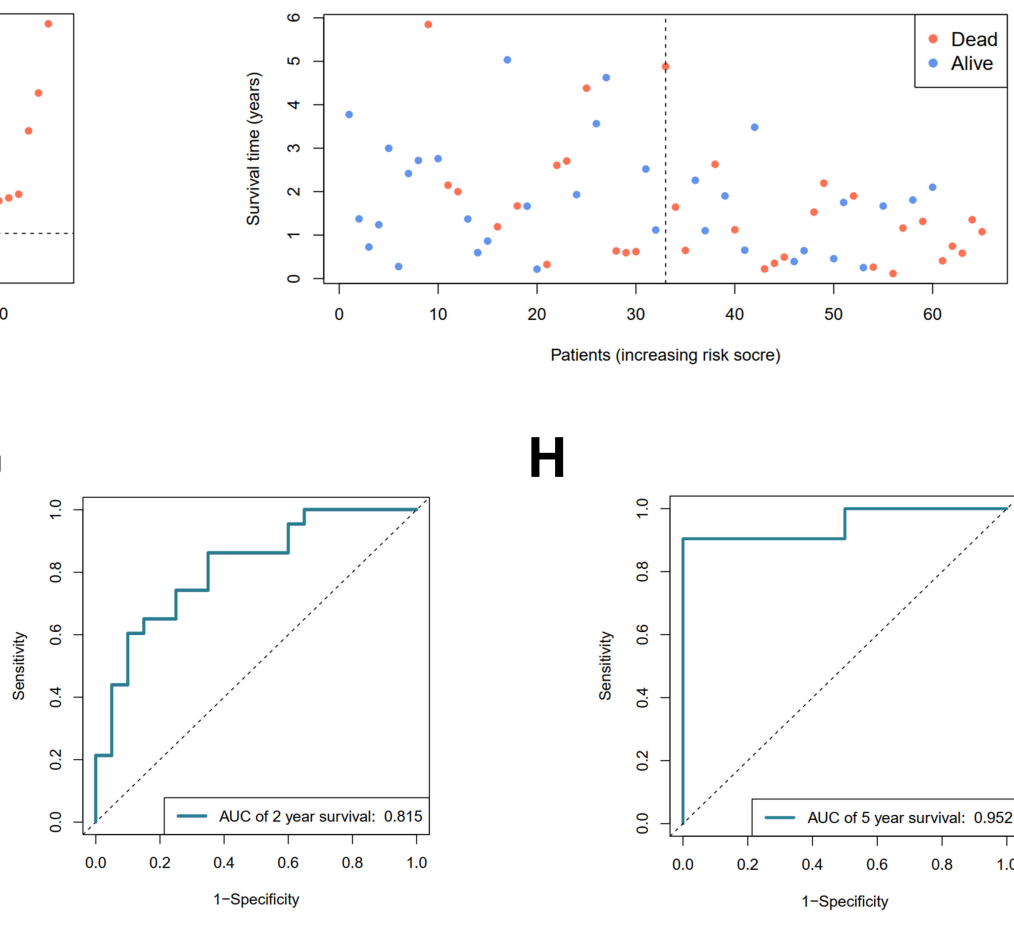

H

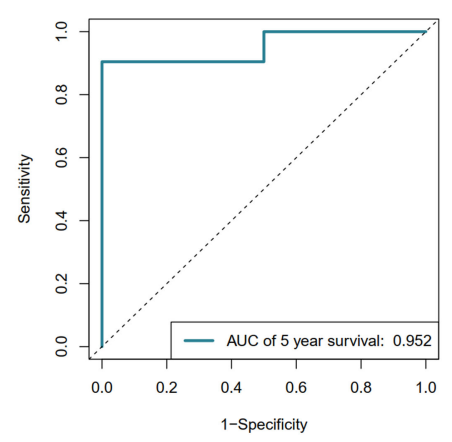

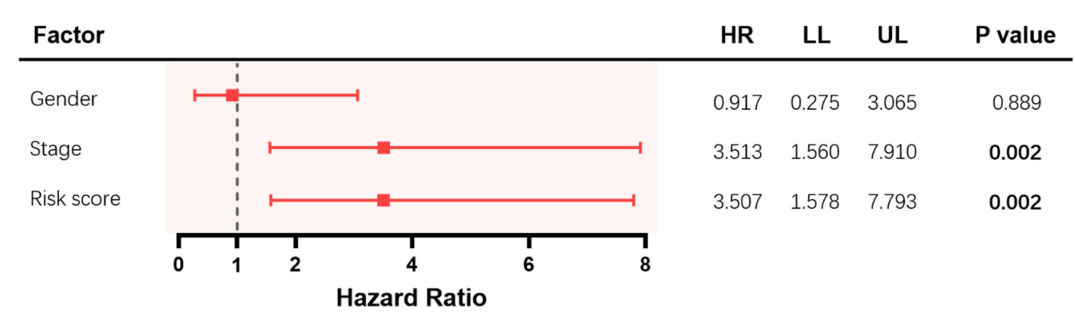

J

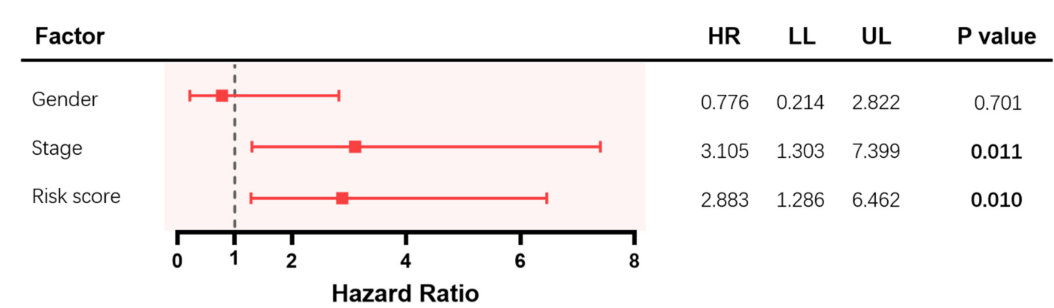


Figure 4

A

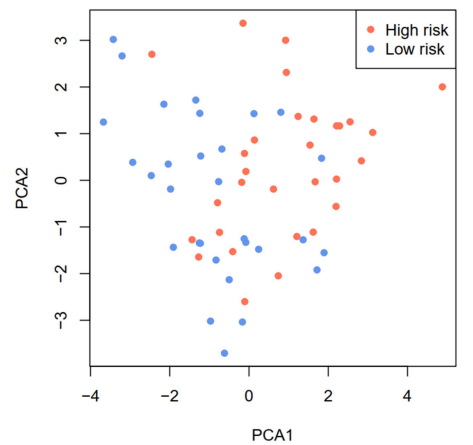

D
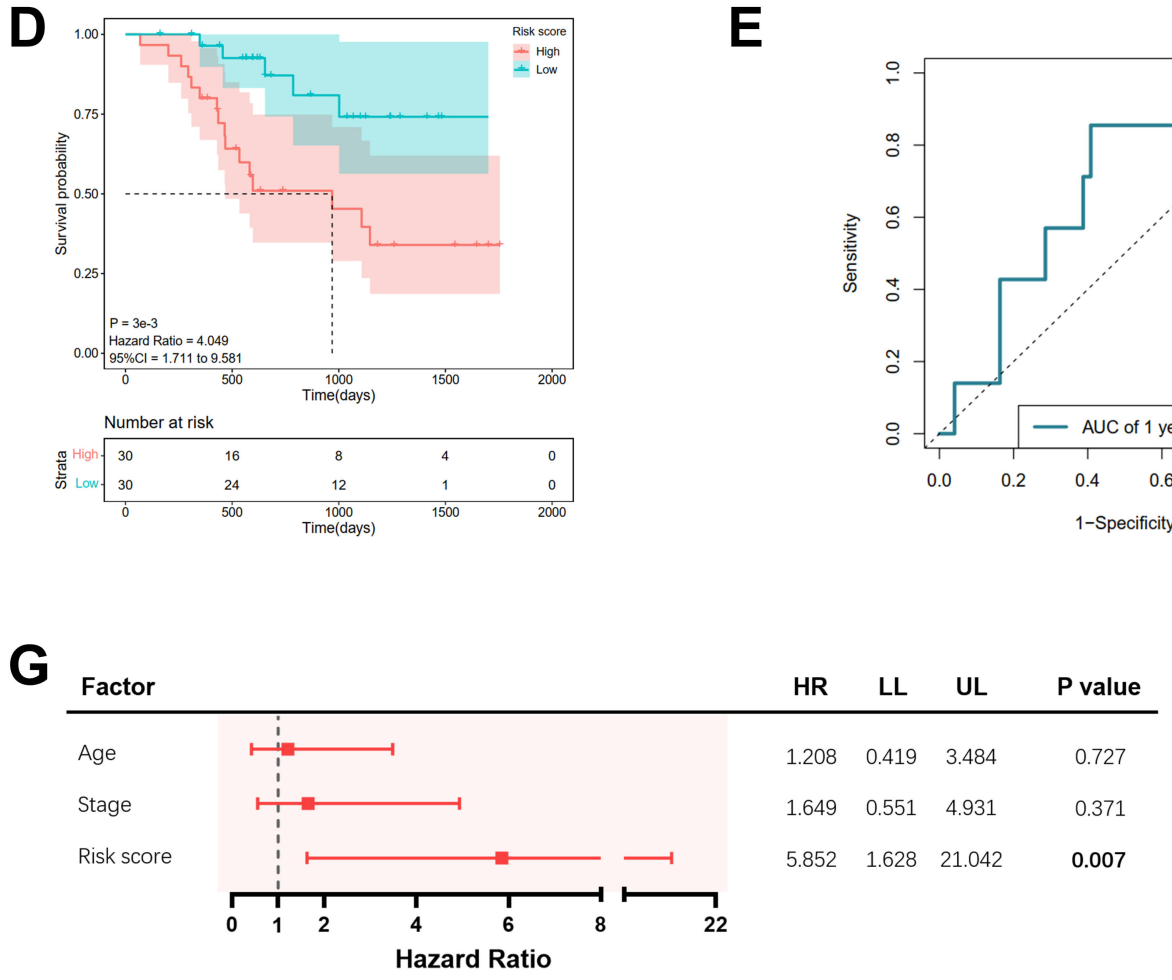

E
C
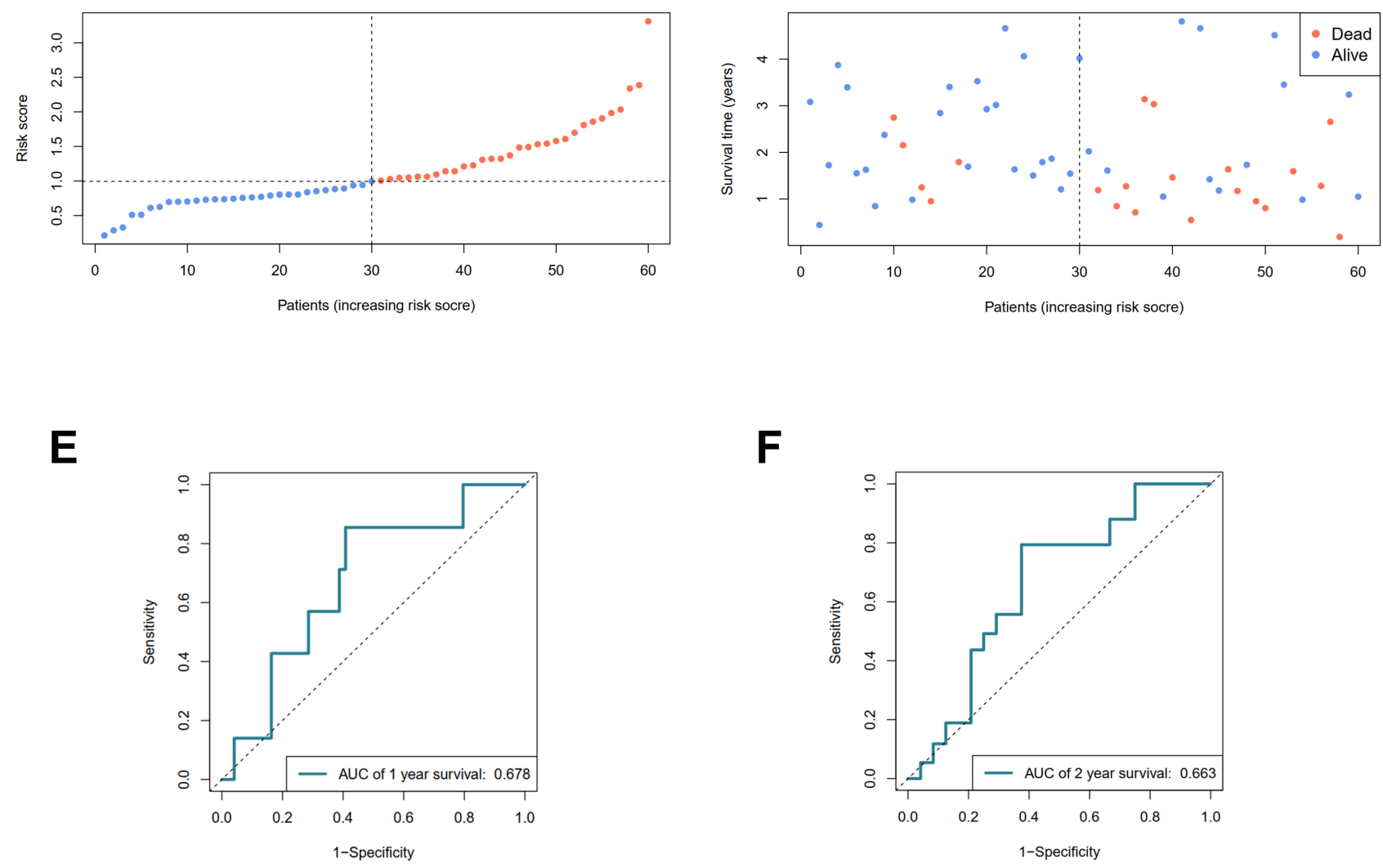

F

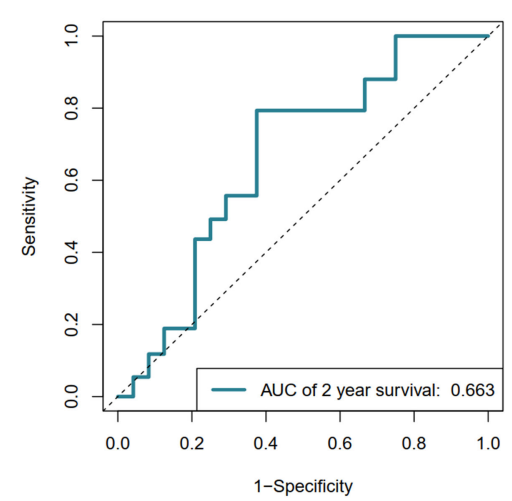

H

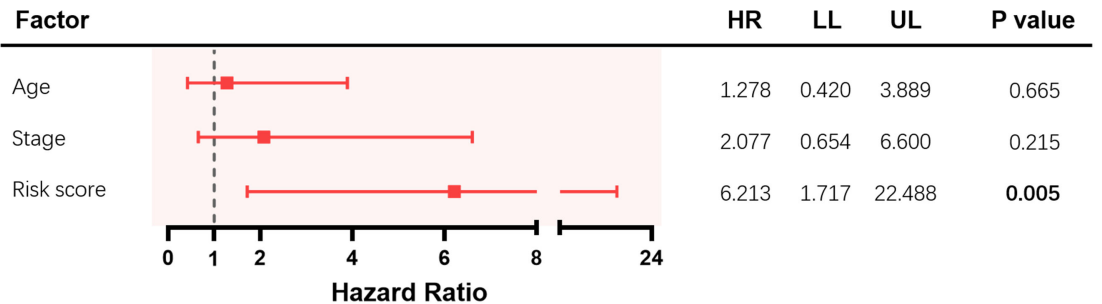


Figure 5

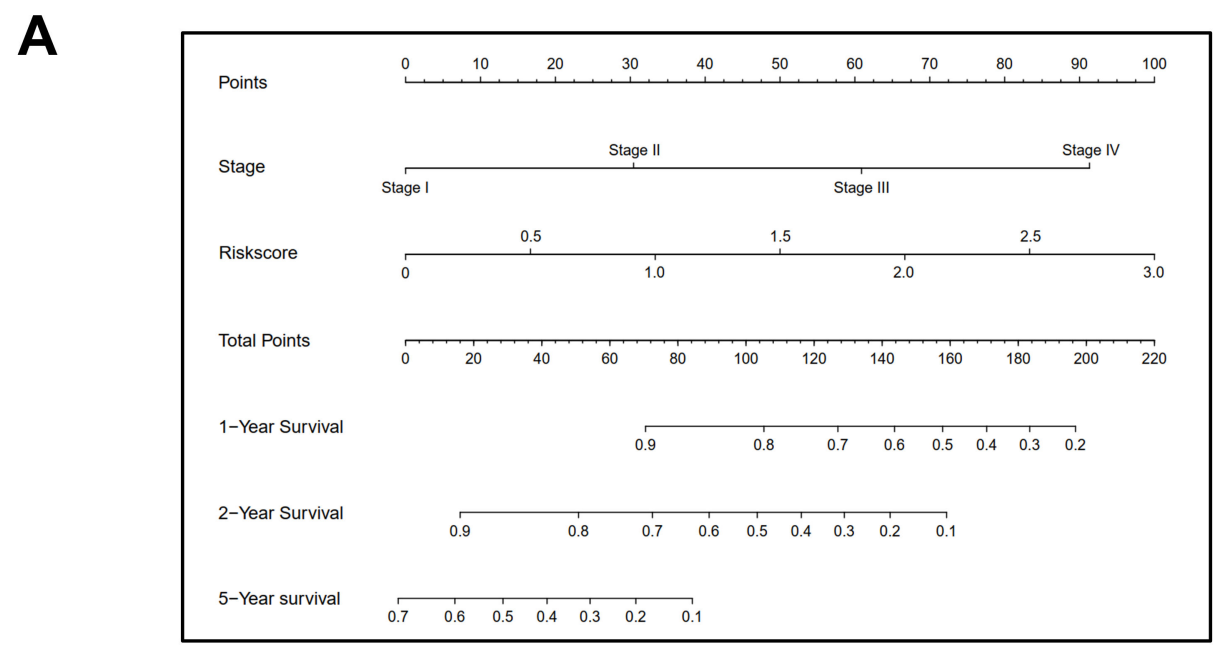

B

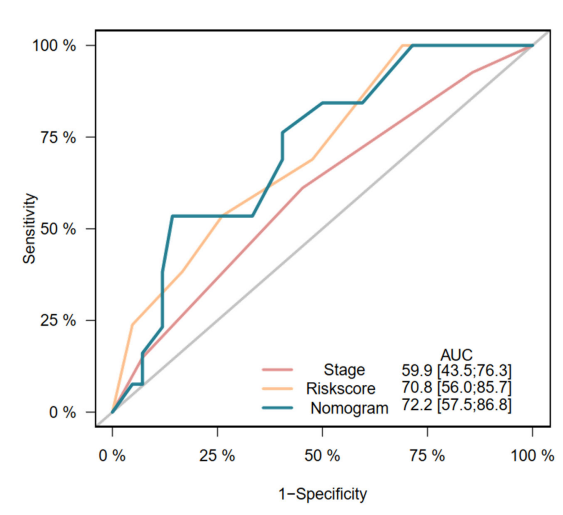

C

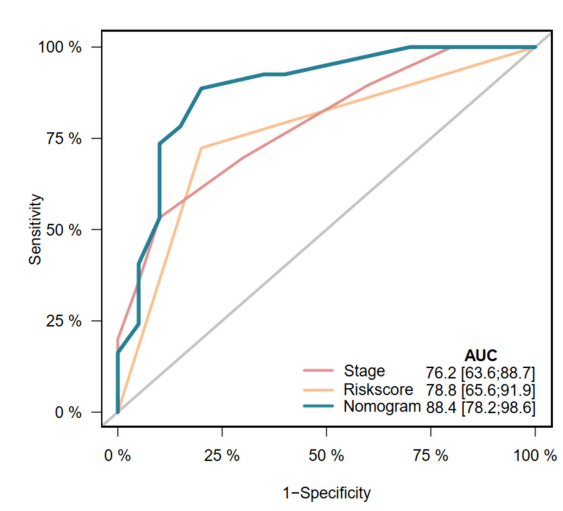

D

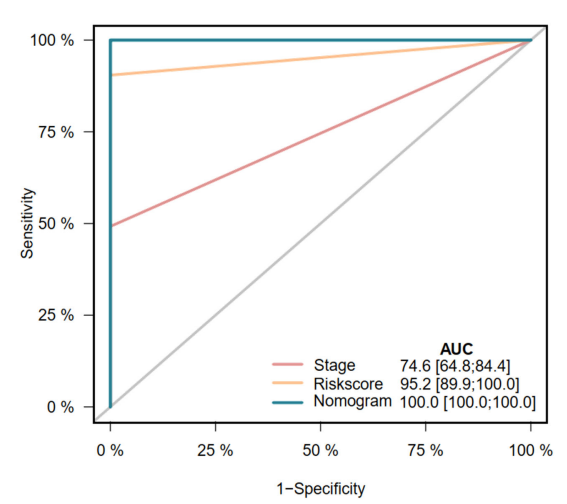

E

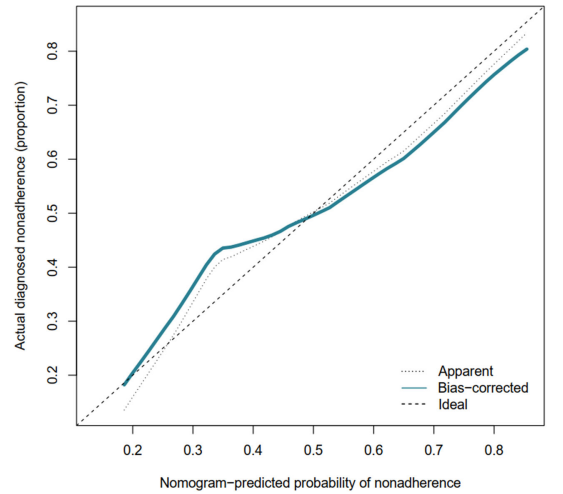

F

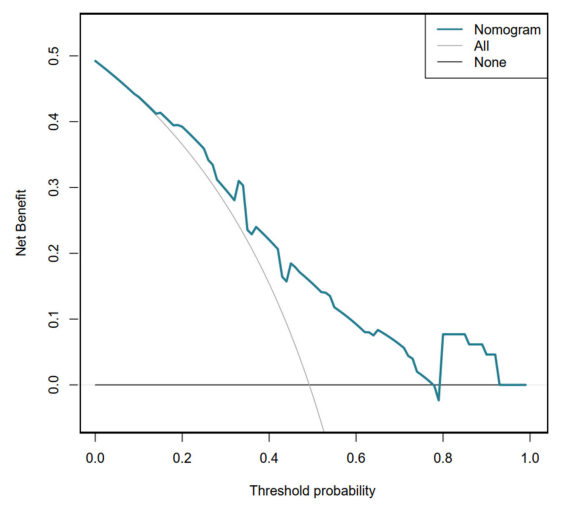


Figure 6

A

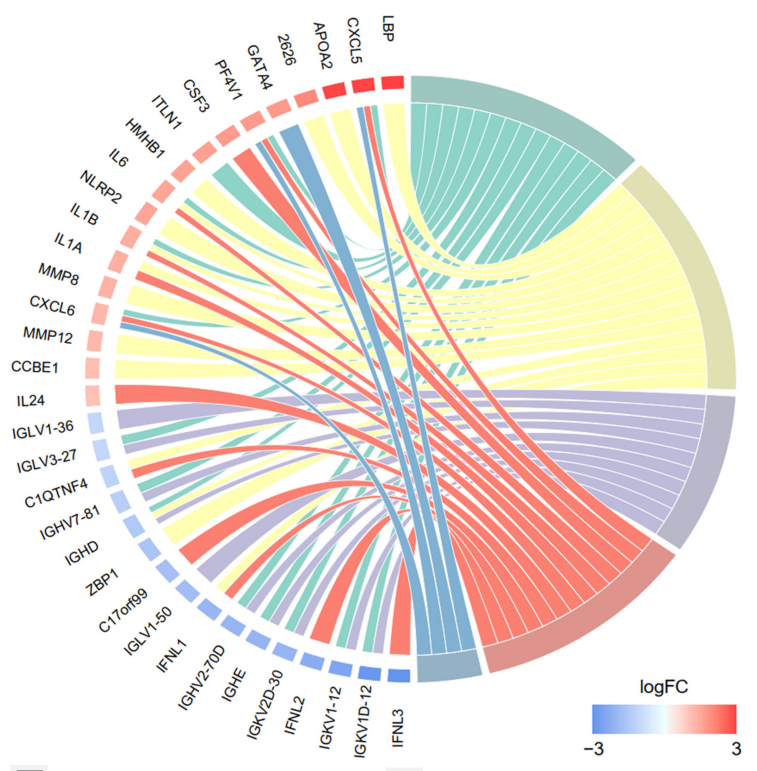

$\square$ Positive regulation of cytokine production $\square$ Immunoglobulin complex

$\square$ Humoral immune response $\square$ Cytokine activity $\square$ cxCR chemokine receptor binding

C

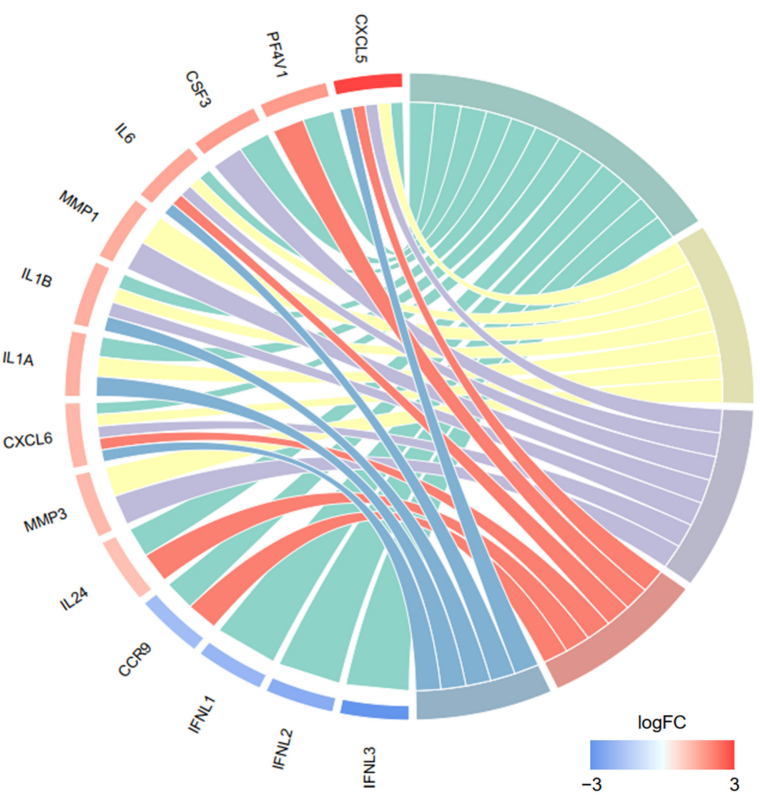

$\square$ Cytokine-cytokine receptor interaction $\square$ IL-17 signaling pathway $\square$ Pertussis

$\square$ Viral protein interaction with cytokine and cytokine receptor $\square$ Rheumatoid arthritis
B

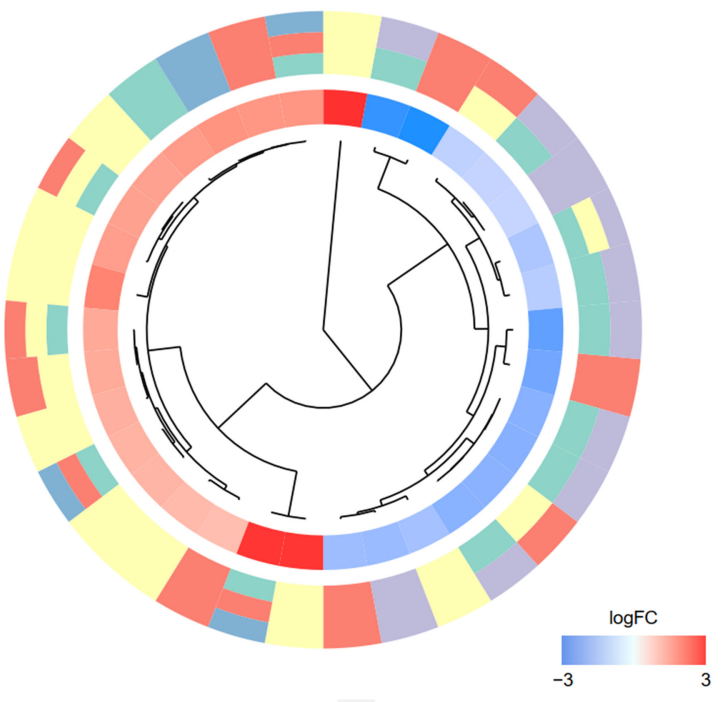

$\square$ Positive regulation of cytokine production $\square$ Immunoglobulin complex

$\square$ Humoral immune response $\square$ cytokine activity $\square$ cxCR chemokine receptor binding

D

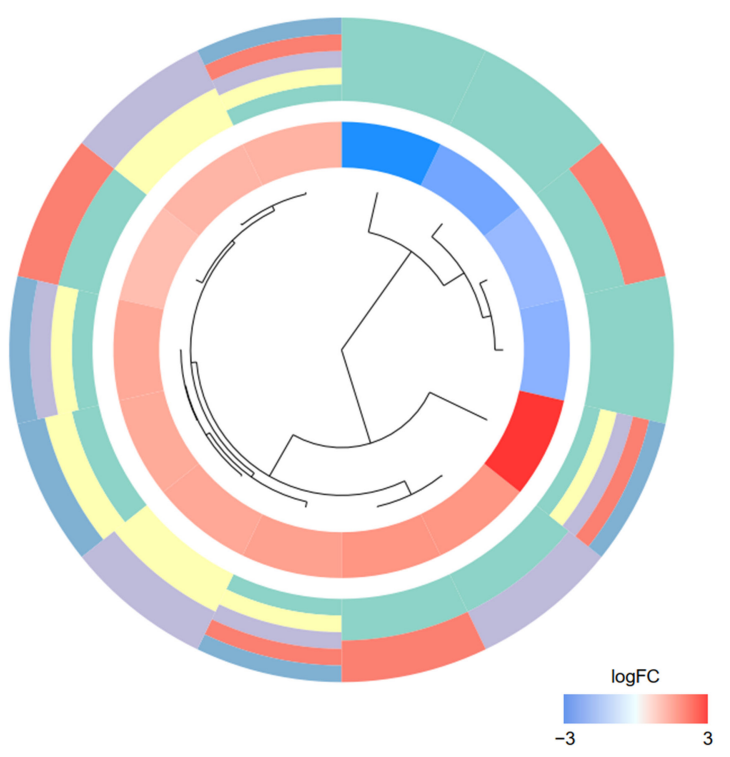

$\square$ Cytokine-cytokine receptor interaction $\square$ L-17 signaling pathway $\square$ Pertussis $\square$ Viral protein interaction with cytokine and cytokine receptor $\square$ Rheumatoid arthritis 
Figure 7

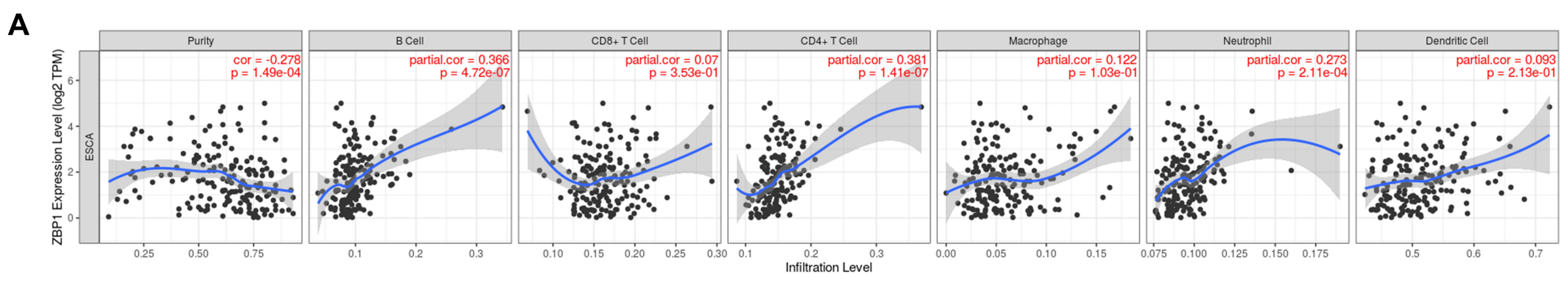

B

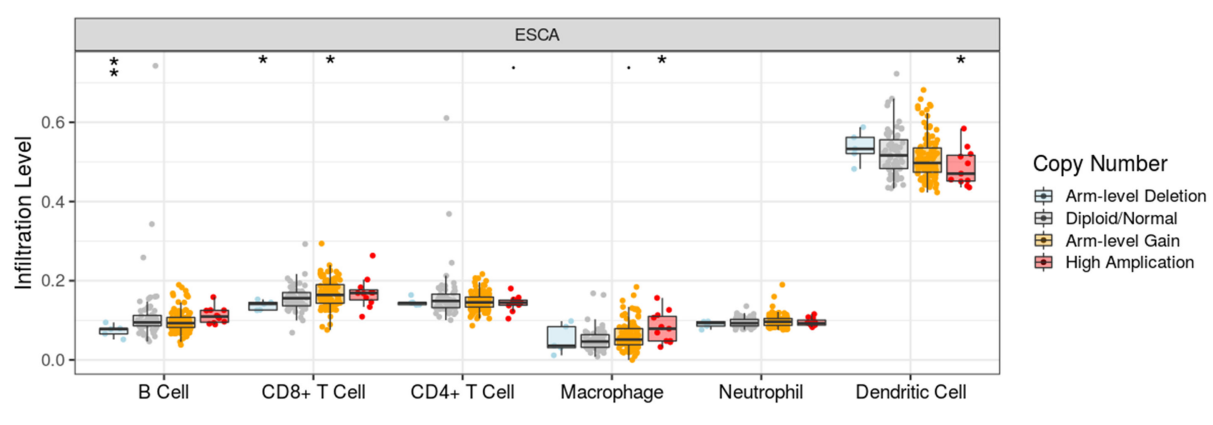


A

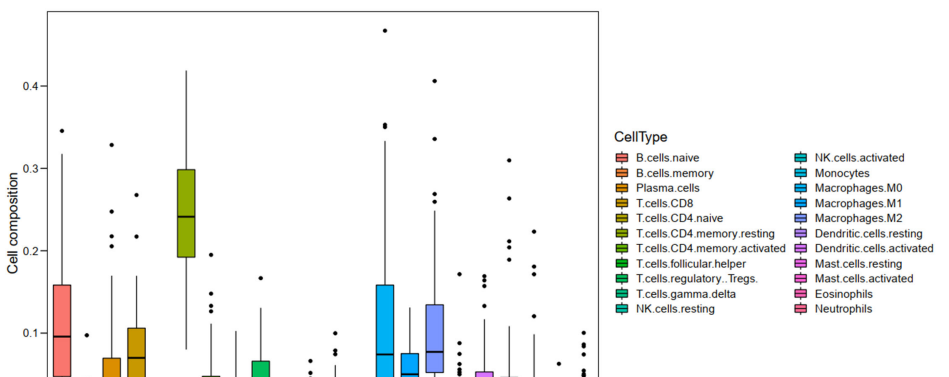

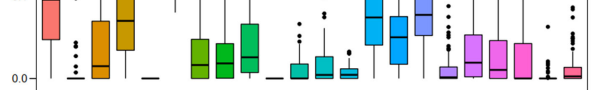
|In|l|||||||||||

c

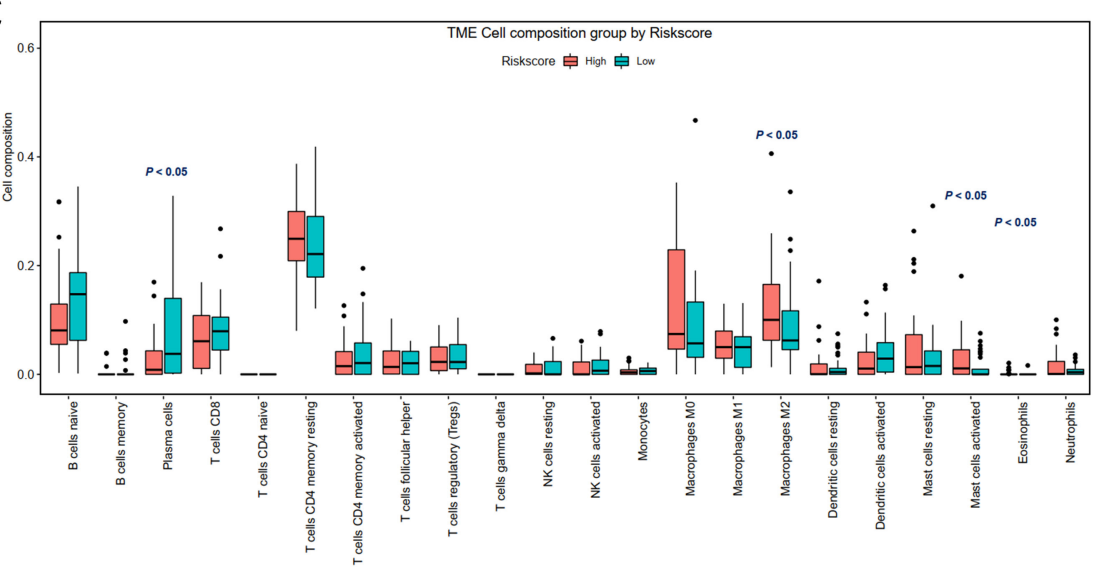

E

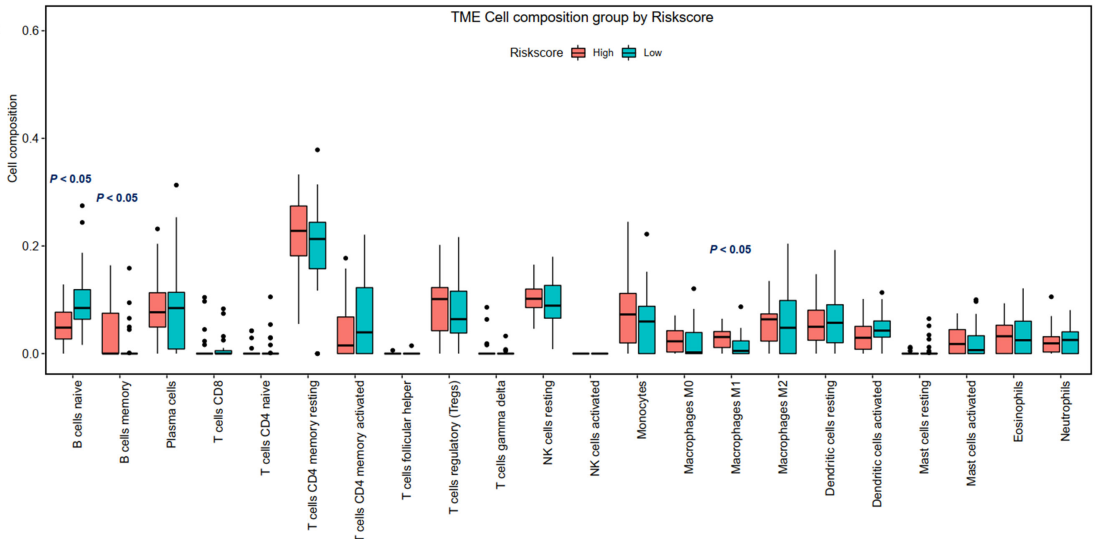

B
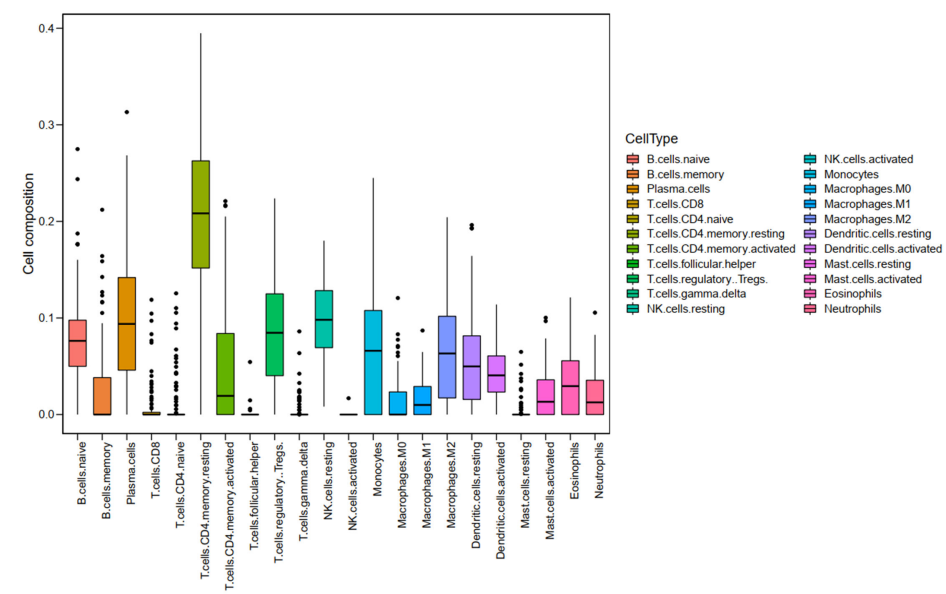

D

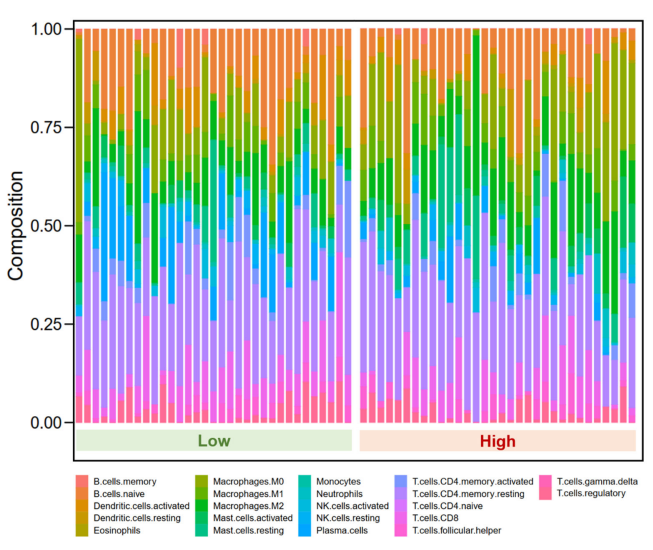

F

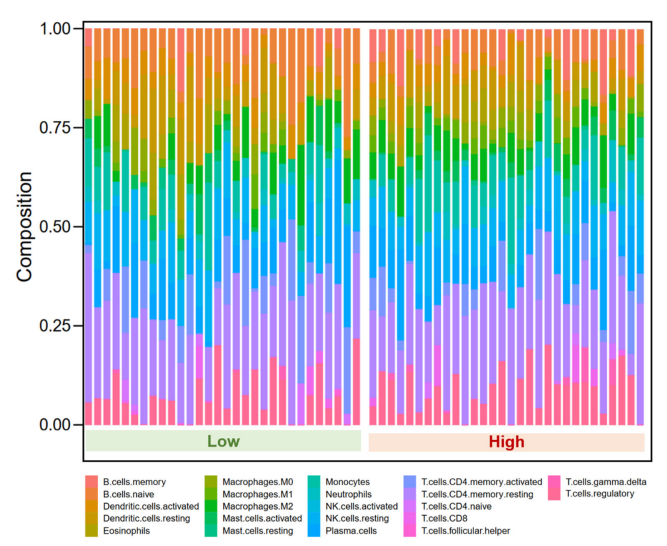

